\title{
Establishment of a duplex RT-PCR assay for simultaneous detection of Rift valley fever virus and peste des petits ruminants virus
}

\author{
Li Guili, Wang Yin, Yao Xueping, Hu Ling \\ Wang Bo, Ren Ranyang, Yang Zexiao ${ }^{a}$ \\ College of Veterinary Medicine, Sichuan Agricultural University, Yaan Sichuan 625014, China \\ aemail:yzxyang2003@126.com
}

Keywords: Rift valley fever virus, Peste des petits ruminants virus, duplex RT-PCR

\begin{abstract}
Rift valley fever(RVF) and peste des petits ruminants(PPR) are both highly contagious diseases of ruminants caused by RVF virus (RVFV) and PPR virus (PPRV), which are both the notifiable multiple species diseases in the OIE list. In order to establish a rapid method for detecting of RVF and PPR, two pairs of primers were designed according to the nucleotide sequence information of RVFV and PPRV published in GenBank. The duplex RT-PCR which contained two pairs of the primers and two templates in one PCR system was established and then the reaction conditions were optimized including temperature and primers concentration. The results showed that the target sequence-products of RVFV and PPRV were 318bp and 589bp, and the duplex RT-PCR assay could be used to detect the RVFV and PPRV with good sensitivity and the detection limit were approximately 53 copies of PPRV and 68 copies of RVFV. Besides, the specificity test proved that there were no cross-reaction with Foot and mouth disease virus, sheep pox virus, E.coli , Salmonella and Pasteurella multocida isolated from ruminants and blood sample of healthy sheep.
\end{abstract}

\section{Introduction}

Rift valley fever (RVF) was first reported to occurred in a farm in Kenya in 1930, and then spread to the African continent and led to millions of dollars of losses[1]. The mainly damage of RVFV is to cause the pregnant ruminants abort and $100 \%$ new born infected-lamb dead. But when the virus infect humans, it cause human chill, fell headache and limb pains, even dead when the disease run to serious stage[2]. RVFV, which belongs to Bunyaviridae family, is an enveloped single-stranded virus[3], and the gene of the RVFV was separate to three segments(L,M and S) to encode different function proteins[4][5][6]. Though the RVFV was payed highly attention by OIE for years, there are no efficient vaccine to prevent the disease no matter in ruminants or humans. With the development of the corporation of the world economy and trade, the risk of the RVFV spreading out of African is increasing including China, though there is no RVFV in China now. So, what we need to do to prevent the disease is to learn it, to establish a rapid detection method and to limit it to enter the country.

Peste des petits ruminants is also a significant disease in the ruminants, which is characterized by fever, sores in the mouth, diarrhoea, pneumonia and even death. PPR was first identified in West Africa in 1940s, and spread out to Africa, Arabia,South East and Asia[7]. PPRV is classified as a member of the Morbillivirus which belongs to the Paramyxoviridae family. More, it is a single-stranded RNA genome which encode eight proteins[8]. PPR was appeared in China since 2007 and led to tremendous losses in breeding industry. Thus, it is necessary to study the virus to avoid the disease keeping on spread.

There are many methods have been designed to detect the two disease separately, like virus isolation, ELISA, RT-PCR and so on[9][10]. RT-PCR is a rapid, high sensitive and already matured method to detect diseases, and multiple PCR was developed based on it. In this study, we established a duplex RT- PCR assay to detect the RVF and PPR simultaneously which infect ruminants seriously. Two pairs of specific primers were designed to amplify the genome of two virus, and the results indicated that the method was an effective tool to detect the two disease. Because there are no RVFV in China and few papers about RVF, we designed the RVFV primers 
based on the synthetic gene fragment of gene S segments by overlapping PCR and the recombinant plasmids were established successfully before and be stored at -20C in Sichuan Agricultural University animal quarantine laboratory[11]. The PPRV gene was collected in PPRV vaccine also provided by Sichuan Agricultural University animal quarantine laboratory.

\section{Materials and Methods}

\section{Reagents and Samples}

DNA Maker DL2000, pMD19-T Vector kitand dNTPs and RNA PCR kit(AMV) were provided by the TaKaRa Biotechnology(Dalian) Co.,Ltd.DH5 $\alpha$ competent cells,2×Taq PCR MasterMix(KT201), Pfu DNA polymerase, TIANprep Mini Plasmid Kit（DP103）, and TIANgel Midi Purification Kit（DP209） were provided by the TIANgen Biotech(Beijing) Co., Ltd, Peste des petits ruminants virus(PPRV) vaccine, Goatpox virus accine, E.coli , Salmonella and Pasteurella multocida isolated from ruminants were provided by Sichuan Agricultural University animal quarantine lab. pMD-19-T-RVFV plasmids was established by overlapping PCR provided by Sichuan Agricultural University animal quarantine laboratory.

\section{Primers}

2 pairs of specific primers was designed by DNAStar according to the sequence data published in Genbank with the segment S of RVFV (accession no:HE687307)and the segment $\mathrm{N}$ of PPRV(accession no:HQ197753.1). And the overlapping PCR primers was also showed in the table 1[11].All of these primers were prepared by the TaKaRa Biotechnology (Dalian) Co., Ltd.

Table1: RVFV and PPRV primers

\begin{tabular}{lcc}
\hline Primer name & \multicolumn{1}{c}{ Sequence (5’-3’) } & Product size \\
\hline RVFV P1 & ATGCACCATCGTCCTAGTCAC & 318bp \\
RVFV P2 & GCAGCTCCTCATTGCTAGAGT & \\
PPRV P1 & ACAAGTTTAGTGCAGGAGC & 589bp \\
PPRV P2 & TGTTGTCTTCTCCCTCCTC & \\
\hline
\end{tabular}

\section{RNA extraction and Reverse transcription}

The RNA of the PPRV was extracted according to the specification. Both of the plasmids and the RNA were stored at -20C. The RNA of the PPRV must reverse transcript to cDNAs before it used to as the templates of the duplex RT-PCR. The reverse transcription would be done with the specification of the RNA PCR Kit (AMV), and the procedure was 37C for $15 \mathrm{~min}$ and $85 \mathrm{C}$ for $5 \mathrm{~s}$. The cDNAs was stored at -20C.

\section{Plasimid construction}

The RVFV plasmids were extracted according to the specification of the TIANprep Mini Plasmid Kit. $20 \mu \mathrm{L}$ reaction system were built for PPRV single RT-PCR with $10 \mu \mathrm{L} 2 \times$ Taq PCR MasterMix, $1.5 \mu \mathrm{L}$ primers(PPRV P1/P2), $2 \mu \mathrm{L}$ plasmids or cDNA templates and $5 \mu \mathrm{L}$ ddH2O. The procedure was 94C for 4min, followed by 35 cycles at 94 Cfor 45s, 55 Cfor 45s, 72C for 1min, and 72 Cfor 10min. $5 \mu \mathrm{L}$ of PCR products were analyzed in a $1.0 \%$ agarose in TAE Buffer gel containing $0.5 \mathrm{mg} / \mathrm{mL}$ GreenView through electrophoresis, which was then photographed under a UV imaging system (Bio-Rad). Correct brands were retrieved by TIANgel Midi Purification Kit and then connected with the pMD19-T vector and transformed into DH5 $\alpha$ competent cells to be amplified. Then, the recombinant plasmids constructed were extracted using a TIANprep Mini Plasmid Kit and were subsequently identified by PCRs (using PPRV P1/P2 and pMD-19-T P1/P2 as primers, respectively) and by sequencing analysis.

\section{Duplex RT-PCR}

On the basis of single RT-PCR, duplex RT-PCR was established quickly with the reaction system were $10 \mu \mathrm{L} 2 \times \mathrm{Taq}$ PCR MasterMix, $1 \mu \mathrm{Lprimers(RVFV} \mathrm{P1/P2}$ and PPRV P1/P2), $1 \mu \mathrm{L}$ templates(RVFV plasmids and PPRV cDNA) and $4 \mu \mathrm{L}$ ddH2O. The procedure was the same with single RT-PCR.

\section{Optimization of the duplex RT-PCR}

In order to obtain the duplex RT-PCR optimization conditions, temperature and primer 
concentration have been optimized. The temperature was optimized under 52C to 59C. $0.1 \mu \mathrm{L}$ to $0.7 \mu \mathrm{L}$ primers separately added to the reactions to optimize the primers concentration. The results would be judged by the image of the band under the UV imaging system.

\section{Specificity and Sensitivity tests}

The specificity of the duplex RT-PCR was assessed through comparing the RVFV plasmids and PPRV cDNAs with cDNA templates of the Foot and mouth disease virus (FMDV) vaccine, DNA templates of sheep pox virus vaccine, E.coli , Salmonella and Pasteurella multocida isolated from ruminants and to a blood sample of sheep. The DNA or cDNA templates were prepared by using the same way with the PPRV in this study. To assess the sensitivity, the recombinant plasmids constructed above were estimated by a ND-1000 ultraviolet spectrophotometer (Nano Drop Co.,Ltd,USA) and were then amplified in a 10-fold serial dilution by RT-PCR using the optimum reaction system and conditions as determined above.

\section{Results}

\section{Plasimid construction}

To obtain the target fragments, the two single RT-PCR were established to amplify the reversed RNA, the results were showed in Fig1. Then, the recombinant plasmid was identified by two types of PCR methods (Fig.2)and sequencing analysis. The PCR products of the recombinant plasmids from the pMD19-T vector usual primers were about $727 \mathrm{bp}$, which were $138 \mathrm{bp}$ longer than the PCR products from the special primers(PPRV P1/P2). And the Blast analysis of the sequencing results of the recombinant plasmids of PPRV revealed that it had a 100\% homology with the reference sequences that are published in GenBank(accession no:HQ197753.1).

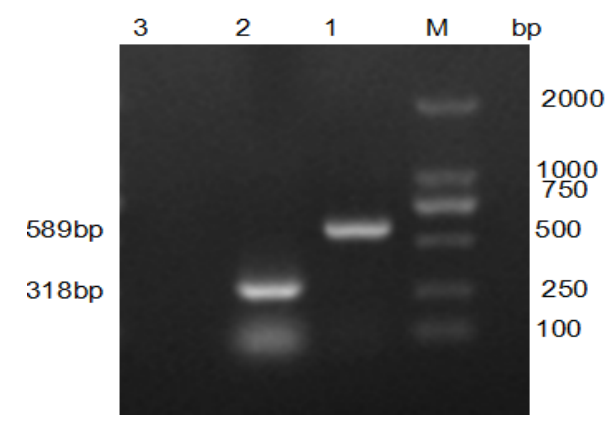

Fig.1. Synthesis result of target DNA fragments. M:DNA marker DL2000. 1: RVFV. 2:PPRV. 3: negative control.

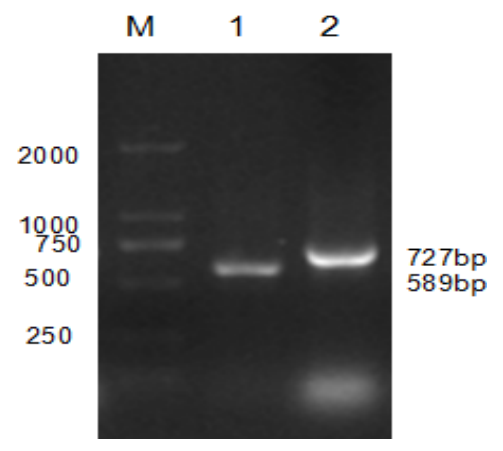

Fig.2. The PCR results of PPRV and recombinant plasimd. M:DNA markerDL2000.1:PPRV. 2:recombinant plasimd.

\section{Duplex RT-PCR}

As we could see in the Fig 3, the two target brands were both amplified in one PCR system. It was proved that the duplex RT-PCR to detect the two ruminants virus were established successfully.

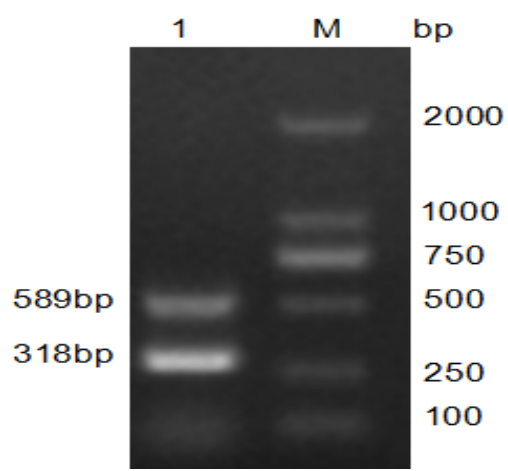

Fig.3. Duplex RT-PCR result M:DNA marker DL2000. 1: RVFV and PPRV 


\section{Optimization of the duplex RT-PCR}

The picture of temperature gradient was showed in the Fig.4. As we could see in the picture, 56C was the optimal temperature in the test. 0.6ul primers were the optimal concentrations in the reaction determined in the Fig.5. So, the final reaction system was 10ul 2×Taq PCR MasterMix, $0.6 \mathrm{ul}$ all the primers, $1 \mathrm{ul}$ both the templates, $5.6 \mathrm{ul} \mathrm{ddH2O}$, and the ultimate reaction procedure was 94C for 4min and 94 Cfor 45s, 56 Cfor 45s, 72C for 1min for 35 cycles, and 72 Cfor 10min.

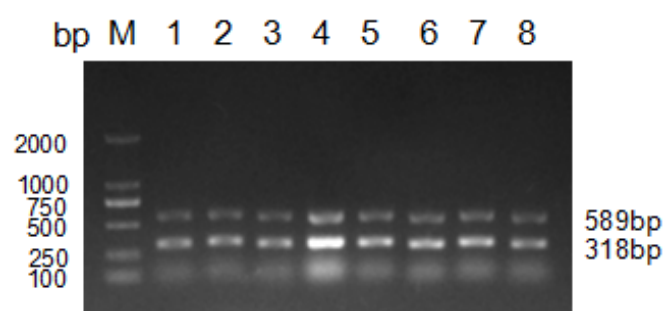

Fig.4. Temperature optimal result. M:DNA marker DL2000. $1: 52^{\circ} \mathrm{C} .2: 53^{\circ} \mathrm{C} \cdot 3: 54^{\circ} \mathrm{C} .4: 55^{\circ} \mathrm{C}$. $5: 56^{\circ} \mathrm{C} .6: 57^{\circ} \mathrm{C} .7: 58^{\circ} \mathrm{C} .8: 59^{\circ} \mathrm{C}$.

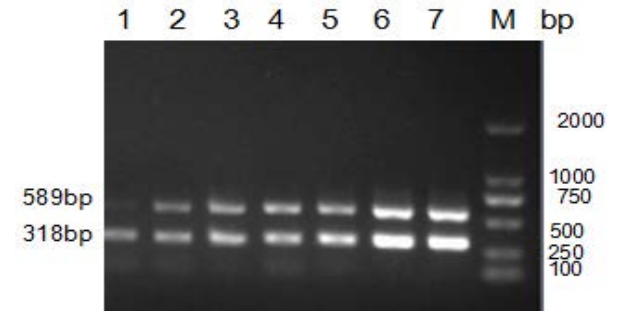

Fig.5. Primers optimal result. M:DNA marker DL2000. 1:0.1ul. 2:0.2ul. 3:0.3ul. 4:0.4ul. 5:0.5ul. 6:0.6ul. 7: $0.7 \mathrm{ul}$.

\section{Specificity and Sensitivity tests}

The specificity test was compared the RVFV and PPRV with FMDV, sheep pox virus, E.coli , Salmonella and Pasteurella multocida isolated from ruminants and blood sample of healthy sheep. The results showed that the RVFV and PPRV had clear brand in 318bp and 589bp but no specific amplification in the other system, which had improved that the duplex RT-PCR was a high specific method to detect the two disease[Fig.6].For the sensitivity of the method, PCR products could be observed when almost 53 copies of PPRV and 68 copies of RVFV target fragments were detected (as shown in Fig.7).

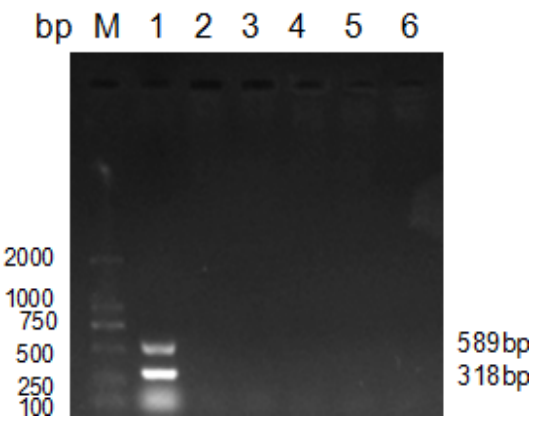

Fig6. The specificity test result. M:DNA marker DL2000. 1:duplex RT-PCR. 2:FMDV. 3:sheep pox virus. 4:E.coli. 5:Pasteurella multocida. 6:blood sample of healthy sheep.

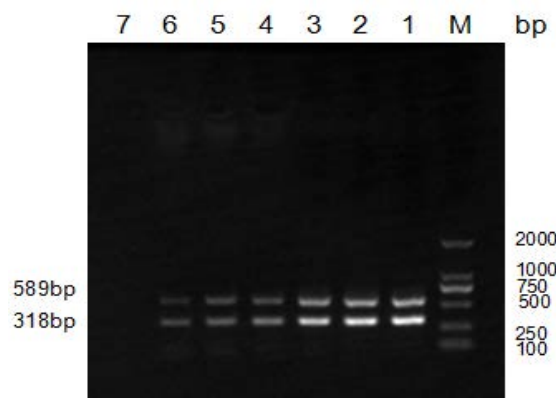

Fig.7:The sensitivity test results of duplex RT-PCR. M: DNA marker DL2000. 1:5.3×106 copies of

PPRV and 6.8 $\times 106$ copies of RVFV. 2:5.3×105 copies of PPRV and 6.8 $\times 105$ copies of RVFV.

3:5.3×104copies of PPRV and 6.8 $\times 104$ copies of RVFV. 4: $5.3 \times 103$ copies of PPRV and $6.8 \times 103$

copies of RVFV. 5: 530 copies of PPRV and 680 of RVFV. 6: 53 copies of PPRV and 68 copies of

RVFV. 7: 5.3 copies of PPRV and 6.8 copies of RVFV. 


\section{Conclusion and Discussion}

At present, various methods have been established to detect the RVFV and the PPRV which are both infected ruminants separately. As we all known, RVF is a very important amphixenosis spread in Africa mainly. But we cannot ignore that RVF was spread by mosquitoes which can be found everywhere, which add the risk of the disease coming out to other countries[12][13] including China. We must watch out for the RVF anytime by establishing an effective system including detection, prevention and controlling to prevent it incoming. Unlike RVF, PPR has occurred in China already in 2007, and there are more studies in PPR and PPRV compared with RVF. PPR's detect assays have been built in all aspects of the virus, and the vaccine of the PPR has been applied to the industry to prevent the disease and obtained favorable effect as well[14], which is not mean that we could stop the step to continue studying PPRV. We need to study more and more until we wipe out the virus on the contrary.

The innovating of this study was to established a high specific and sensitive assay to detect the two diseases simultaneously and it was the first time to establish an assay to detect the two diseases simultaneously in China. As is well-known to all, RVFV and PPRV could infect ruminants like sheep and cattle. When the animals infected virus, we could save the time to distinguish with the disease in the clinically by using this assay. In addition, what we have done in this study is that a rapid, specific and sensitive duplex RT-PCR had been built to detect the two significant diseases in ruminants. And we wish that what we have done could provide some reference in two diseases prevention and control.

\section{Acknowledgements}

This study was supported by Grants from the planning subject of 'the twelfth five-year-plan' in national science and technology for the rural development in China(2013BAD12B04), and “211-Projects” Shuangzhi Plan in Sichuan Agricultural University. Wang Yin should also be considered as first authors. Corresponding author: Yang Zexiao

\section{References}

[1] Caroline AL, Powell DS, Bethel LM, Oury TD, Reed DS, Hartman AL.Broad spectrum antiviral activity of favipiravir (T-705): protection from highly lethal inhalational rift valley Fever[J]. PLoS Negl Trop Dis. 2014, 8(4):e2790.

[2]Jeroen Kortekaas.One Health approach to Rift Valley fever vaccine development[J]. Antiviral Research,2014,106:24-32.

[3]Turell, M.J., Linthicum, K.J., Patrican, L.A., Davies, F.G., Kairo, A., Bailey,C.L. Vector competence of selected African mosquito(Diptera:Culicidae) species for Rift Valley fever virus[J]. J. Med. Entomol. 2008. 45:102-108.

[4] Gerrard S R,Nichol S T,Synthesis, proteolytic processing and complex formation of N-terminally nested precursor proteins of the Rift Valley fever virus glycoproteins[J]. Virology,2007,357:124-133.

[5]Won S Ikegami T, peters C J ,et al. NSm and 78-kilodalton proteins of Rift Valley fever virus are nonessential for viral replication in cell culture[J]. J Virol ,2006,80:8274-8278.

[6] Le May N, Gauliard N, Billecocq A,et al. The N terminus of Rift Valley fever virus mucleoprotein is essential for dimerization[J]. J Virol, 2005,79:11974-11980.

[7] Dhar, P., Sreenivasa, B.P., Barrett, T., Corteyn, M., Singh, R.P., Bandyopadhyay, S.K., Recent epidemiology of peste des petits ruminants virus (PPRV)[J]. Vet. Micro-biol.2002, 88, 153-159.

[8] Bailey, D., Banyard, A.C., Dash, P., Ozkul, A., Barrett, T., Full genome sequenceof peste des petits ruminants virus, a member of the Morbillivirus genus[J]. VirusRes. 2005,110, 119-124. 
[9]Shall A A,Thomnon J, Sene O K, et al, Single-tube and nested reverse transcriptase-polymerase chain reaction for detection of Rift Valley fever virus in human and animal sera[J]. J Virol Methods, 2001,91:85-92.

[10]Bao J, Li L, Wang z, et a1. Development of one-step real time RT-PCR assay for detection and quantitation of peste des petits ruminants virus[J]. J Virol Methods 。 2008. 148(1-2): 232-236.

[11]Yang Zexiao, Wang Bo, Xu Qiumei,et al.Design and Evaluation of the Primers for Rift Valley Fever ( RVF ) Virus RT- PCR Detection[J].Advanced Materials Research Vols.2014,(989-994):1115-1119.

[12]Turell M J, Dohm D J, Mores C N。 el a1. Potential for North American mosquitoes to transmit Rift Valley fever vires[J]. J Am Mosq Control Assoc, 2008, 24: 502-507.

[13]Iranpour M, Turell M J, Lindsay L R. Potential for Canadian mosquitoes to transmit Riii Valley fever virus[J]. J Am Mosq Control Assoc, 2011. 27: 363-369.

[14]Sen A. Balamurugan V. Rajak K K, et a1. Role of heavy water in biological sciences with an emphasis on thermostabilization of vaccines[J]. Expert Rev Vaccines. 2009, 8: 1587-1602. 